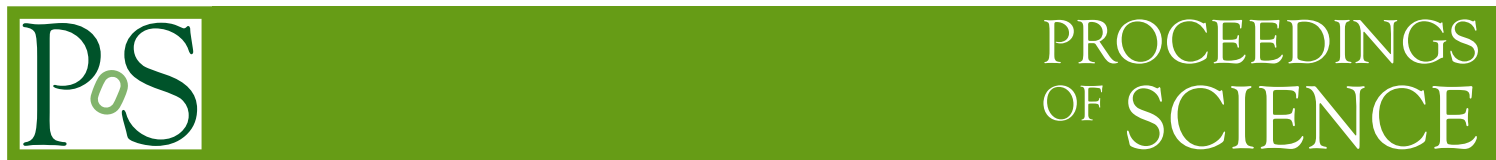

\title{
Jet-medium interactions in heavy ion collisions
}

\author{
Olga Evdokimov* for the STAR Collaboration \\ University of Illinois at Chicago \\ E-mail: evdolga@uic.edu
}

\begin{abstract}
Angular correlation studies at RHIC have presented evidences of strong interactions between hard partons and the QCD matter, providing experimental constrains on medium properties, jet fragmentation and theoretical models of energy loss. Those early tools for studies of jet-medium interactions in heavy ion collisions have recently been expanded by addition of full jet reconstruction techniques, providing a more direct measurement of the initial parton energy, thereby further advancing our knowledge of jet-medium interactions. In this paper we present a brief overview of new STAR results for jet quenching via triggered correlations along with the first measurements from jet reconstruction in $\mathrm{Au}+\mathrm{Au}$ collisions.
\end{abstract}

35th International Conference of High Energy Physics - ICHEP2010,

July 22-28, 2010

Paris France

* Speaker. 
One of the most remarkable discoveries of the ongoing heavy ion physics program at RHIC is the experimental observation of the jet quenching [1]. The first experimental evidence for jet quenching discovery came from the studies of relative production rates of the high transverse momenta $\left(p_{T}\right)$ hadrons in $\mathrm{Au}+\mathrm{Au}$ collisions compared to $\mathrm{p}+\mathrm{p}$ events at the same center-of-mass energy. The strong suppression observed is attributed to the parton energy loss in the created medium $[2,3]$, however, studies of the inclusive hadron distribution have limited power in discrimination of different energy loss scenarios or quantitative measurements of parton energy loss. The idea of "jet tomography", studies of the medium properties via hard scattered partons, has been a tempting endeavor for the heavy ion physics since the beginning of RHIC era. The main obstacle for such studies in A+A events is large multiplicity of the final state produced.

Until recently the major tool for jet studies was 2- and 3-particle angular correlations. The STAR detector, with its extended coverage and nearly uniform acceptance, is ideally equipped for such analysis. Despite inability to measure directly the parton energy, these studies uncovered large set of novel features. The jet-like peaks observed in the angular correlations of hard hadron trigger and soft associated hadrons [4] show significant shape differences between Au+Au and p+p measurements. The comparisons of spectral distributions for hadrons, associated with the high $p_{T}$ trigger on away-side $(\Delta \phi \approx \pi)$, in different centrality bins of $\mathrm{Au}+\mathrm{Au}$ events exhibit progressively softer $p_{T}$-spectrum compared to that in $\mathrm{p}+\mathrm{p}$ data. At higher transverse momenta $\left(1.0<p_{T}<2.0 \mathrm{GeV} / c\right)$ in addition to the broadening the development of a dip at $\pi$ is observed [5, 6]. Modifications of correlation structures are also evident on a same-side (small $\Delta \phi$ ) of a trigger particle. Recent studies [7] of azimuthal distributions of hadrons about high- $p_{T}$ trigger yielded an observation of a novel long-range correlation structure (the "ridge") in the relative pseudo-rapidity, not seen in $\mathrm{p}+\mathrm{p}$ or d+Au data from RHIC. Similar observations were seen in the measurements of untriggered angular pair-correlation in the soft sector [8]. Jet-like features observed suggest that non-thermal hadron emission in this sector affects significant fraction of hadrons (10-30\%) event in most central collisions. Theoretical interpretations of these features are non-trivial. Various mechanisms proposed to accommodate experimental findings, including shock wave induced conical flow (Mach-cone), gluon radiation, hadronization via quark recombination, CGC color-flux tubes and others (see refs. in $[4,6]$. Some of the difficulties could be attributed to the trigger effects (dilution, surface bias, etc.) and ambiguity of signal and background separation in the two-component approach used in the above works. Below we discuss a few new developments that attempt in different ways addressing (or controlling) those biases.

The idea behind the next approach to study in-medium energy deposition by the hard-scattered parton is to look at the energy balance between the di-jets reconstructed via " $2+1$ " three-particle correlation technique. In the analysis two high- $p_{T}$ back-to-back hadrons are used to define the di-jet axis, and then associated hadron distribution in $\Delta \eta$ and $\Delta \phi$ space is studied simultaneously on the same- and away-side about each respective trigger. The trigger pair is selected such that the primary (first) trigger is the highest $p_{T}$ particle in the event, insuring the most surface bias for the same-side jet, while the away-side trigger threshold can be varied to probe different pathlengths. With nearly symmetric trigger combinations $\left(p_{T}^{\text {trig1 }}>5 \mathrm{GeV} / c, p_{T}^{\text {trig } 2}>4 \mathrm{GeV} / c\right)$ it has been shown (see Fig. 1) that extracted correlation signal on both sides of di-jet-like pair is consistent with in-vacuum jet fragmentation, as no modifications are apparent in either shape or strength of the correlation between heavy ion and $\mathrm{d}+\mathrm{Au}$ data [9]. The transverse momentum distribution of 
the associated hadrons are also consistent between all centrality bins of $\mathrm{Au}+\mathrm{Au}$ data and $\mathrm{d}+\mathrm{Au}$ collisions. The production rates measured for such di-jets with near-symmetric trigger conditions suggest tangential emission scenario, with geometry of the interaction zone playing a major role in suppression. Alternative explanation could be provided by the absence of interactions with the hot and dense medium for such di-jets, but the production rates observed are yet to be explored in such models. Selection of a back-to-back pairs for a di-jet trigger allows removal of non-jet contributions and trigger-dilution effects, providing cleaner probe for jet-medium interactions compared to the di-hadron correlations. Studies are under way for the different kinematics (asymmetric) trigger pairs [10], with precise measurements expected from higher statistics data samples projected in coming years.
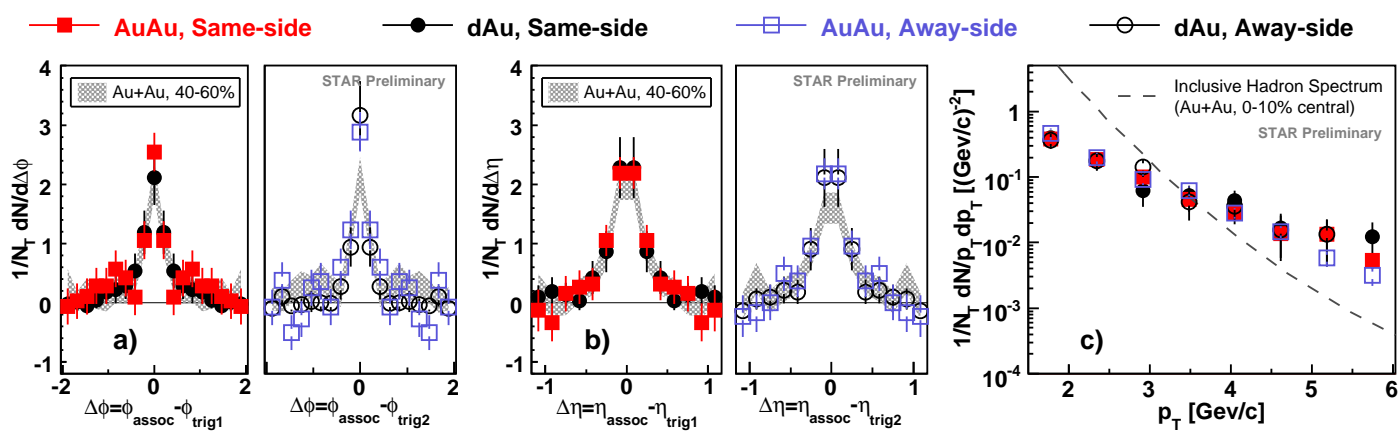

Figure 1: Di-jet triggered three particle correlation results from STAR $200 \mathrm{GeV}$ data. $\Delta \phi(a)$ and $\Delta \eta(b)$ projections are shown for the following kinematic selection: $5<p_{T}^{\text {trig1 }}<10 \mathrm{GeV} / c, 4 \mathrm{GeV} / c<p_{T}^{\text {trig2 }}<p_{T}^{\text {trig1 }}$, $1.5<p_{T}^{a s s o c}<p_{T}^{\text {trig } 1} \mathrm{GeV} / c . c$ ) Transverse momentum spectral distributions for the same- and away-side hadrons $(\Delta \phi<0.5, \Delta \eta<0.5)$.

Direct gamma measurements have potential to provide access to the initial parton energy without fragmentation-related uncertainties of hadron studies. The dominant channels of $\gamma_{\text {dir }}$ production are quark-gluon Compton scattering and quark-antiquark annihilation. In both processes outgoing photon, which is not expected to interact with the medium, is balanced by the jet. The details of this balance (or deviations from such in $\mathrm{Au}+\mathrm{Au}$ comparatively to $\mathrm{p}+\mathrm{p}$ collisions), can be studied with direct gamma-hadron correlations. In STAR the Barrel Electromagnetic Calorimeter (BEMC) was used for $\gamma_{d i r}$ and neutral pion $\pi^{0}$ reconstruction; analysis of the shower-shapes allowed initial discrimination between the two. Remaining background was subtracted assuming no correlated hadron production on the near side of the true direct photon. Azimuthal correlations with the charged hadrons measured in central $\mathrm{Au}+\mathrm{Au}$ collisions at mid-rapidity have shown large suppression of the away-side yields with respect to both types of triggers as compared to the binary scaled $p+p$ results. Moreover, within the experimental uncertainties, the suppression level is found to be similar for $\gamma_{d i r}$ and $\pi^{0}$ triggers in the kinematic selection studied (trigger particles with $8<p_{T}^{\text {trig }}<16 \mathrm{GeV} / c$ and associated hadrons with $3<p_{T}^{\text {assoc }}<16 \mathrm{GeV} / c$ ). Figure 2 illustrates this observation: the $I_{A A}$ (binary scaled spectra ratio between $\mathrm{A}+\mathrm{A}$ and $\mathrm{p}+\mathrm{p}$ data for associated hadrons) for direct photon triggers is consistent within errors with that of neutral pions. Statistical limitations for this analysis can be overcome in the coming years with new precision measurements at RHIC II luminosities.

In high energy physics experiments jets are long used to measure the partonic energy. A 


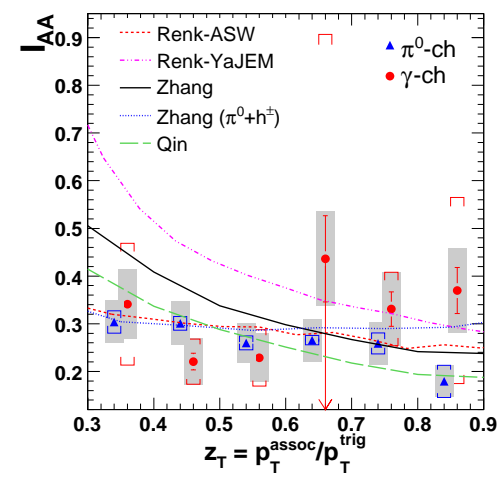

Figure 2: Away-side $I_{A A}$ for $\gamma_{d i r}$ (circles) and $\pi^{0}$-triggers (triangles). For details on the uncertainties shown and theory curves see [11].

major break-through has been recently made by STAR in successfully performing full jet reconstruction for heavy ion events [12]. This required algorithmic developments and novel background subtraction techniques. Jet reconstruction algorithms with a finite jet resolution parameter $\left(R=\sqrt{\Delta \phi^{2}+\Delta \eta^{2}}\right)$ would inevitably bias reconstructed jet population towards less-modified sample, yet evolution of the energy flow can be studied by varying $R$. It has been shown [13] that the ratio of the reconstructed jet spectra is different between $\mathrm{p}+\mathrm{p}$ and $\mathrm{Au}+\mathrm{Au}$ data for the two resolution parameters $(\mathrm{R}=0.2$ over $\mathrm{R}=0.4$ ) studied. In $\mathrm{p}+\mathrm{p}$ data the ratio raises with jet energy towards 1 , consistent with pQCD expectations. The same ratio in central $\mathrm{Au}+\mathrm{Au}$ data is significantly suppressed with weak to no energy dependence, indicating significant change in the jet energy flow. These differences also indicate that even with the large cone radius $(\mathrm{R}=0.4)$, part of the jet energy is missing. Further increase of the resolution parameter is not yet proven practical due to uncertainties caused by background fluctuations. To assess the extent of the jet broadening for heavy ion data and to explore the effects of the medium on the away-side jet, jet reconstruction analysis was expanded by the jet-hadron correlations as done in [15]. The analysis uses anti- $k_{T}$ jet finding algorithm provided by FastJet package [16] with the minimal jet energy requirement of $20 \mathrm{GeV}$ and only hadrons with $p_{T}$ greater than $2 \mathrm{GeV} / c$ included. The $\Delta \phi$ correlation is then constructed for all other charged hadrons in the event with respect to the axis of the jet found. Same-side correlation comparison for $200 \mathrm{GeV} \mathrm{Au}+\mathrm{Au}$ and $\mathrm{p}+\mathrm{p}$ data confirms the broadening of the jet energy flow in soft hadrons. The away-side jet-hadron correlation evolves with $p_{T}^{a s s o c}$ from broadened peak in low momenta to the widths consistent with $\mathrm{p}+\mathrm{p}$ jets in the hard sector (Fig. $3 a$ ). The $p_{T}^{a s s o c}$ dependence of the associated hadron yield in the away-side peak is summarized in the right panel of the Fig. 3, showing the binary scaled ratio of the associated hadron spectra from $\mathrm{Au}+\mathrm{Au}$ and $\mathrm{p}+\mathrm{p}$ events. These observations are consistent with the partonic energy loss, where "missing" energy appears via soft(er) hadrons, partially preserving the correlation with initial parton direction. This picture also explains results of the di-jet coincidence rates measurements in heavy ion collisions [14]. Combining per-event full jet reconstruction with the statistical power of correlation analysis this new analysis provides the means to recover large fraction of initial parton energy and carry out systematic measurements of partonic energy loss.

The experimental approach to jet quenching studies have evolved from single (inclusive) high- 

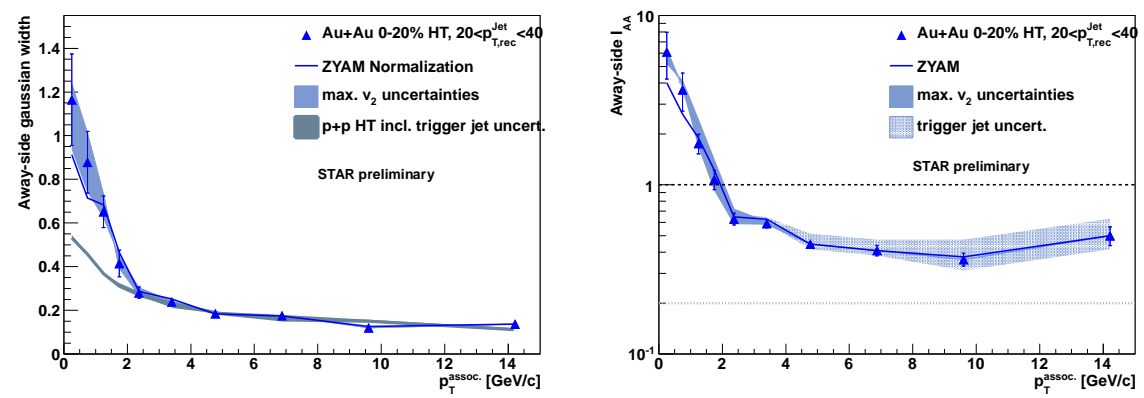

Figure 3: $a$ ) The width of the away-side peak of the jet-hadron correlation from central Au+Au and minimum bias p+p collisions at $200 \mathrm{GeV} ; b$ ) Away-side $I_{A A}$ for hadrons associated with the fully reconstructed jet with $E_{T}>20 \mathrm{GeV}$.

$p_{T}$ hadron measurements to di-hadron and multi-particle angular correlations, and, recently, to full jet reconstruction in heavy ion collisions. The overall consistent picture emerging from the studies: strong jet attenuation leads to the depletion of the high- $p_{T}$ hadrons, the production of the remaining part is likely biased towards the surface. Jet-medium interactions result in the broadening of the jet energy flow; energy lost by the medium traversing parton is redistributed to the soft hadrons preserving partially correlation with the original parton direction. The full potential of the new measurements described here is expected to be reached with analysis of the higher statistics data anticipated in coming years. However, disentangling of the complex experimental biases is needed to deeper understanding of jet quenching phenomenon.

\section{References}

[1] C. Adler et al., Phys. Rev. Lett. 89 (2002) 202301; C. Adler et al., Phys. Rev. Lett. 90 (2003) 082302.

[2] I. Vitev and M. Gyulassy, Phys. Rev. Lett. 89 (2002) 252301.

[3] X. N. Wang, Phys. Lett. $B 595$ (2004) 165.

[4] J. Adams et al., Phys. Rev. Lett. 95 (2005) 152301.

[5] M. M. Aggarwal et al., Phys. Rev. C 82 (2010) 24912.

[6] B. Abelev et al., Phys. Rev. Lett. 102 (2009) 052302.

[7] B. Abelev et al., Phys. Rev. C80 (2009) 064912.

[8] D. Kettler et al., Eur. Phys. J. C62 (2009) 175.

[9] O. Barannikova et al., J. Phys. G 35 (2008) 104086.

[10] K. Kauder et al., Nucl. Phys. A 830 (2009) 685c.

[11] B. I. Abelev et al., Phys. Rev. C 82 (2010) 034909.

[12] S. Salur et al., Eur.Phys.J. C61 (2009) 761c.

[13] M. Ploskon et al., Nucl. Phys. A 830 (2009) 255c.

[14] E. Bruna et al., Nucl.Phys. A830 (2009) 267c.

[15] J. Putschke et al., proceedings of Hard Probes 2010.

[16] M. Cacciari and G. P. Salam, Phys. Lett. B 659 (2008) 119. 